\title{
Calibration analyses and Efficiency Studies for the Anti Coincidence Detector on the Fermi Gamma Ray Space Telescope
}

\author{
Chris Kachulis \\ Office of Science, Science Undergraduate Laboratory Internship (SULI) \\ Yale University \\ Stanford Linear Accelerator Center \\ Stanford, CA
}

August 13, 2010

Prepared in partial fulfillment of the requirements of the Office of Science, Department of Energy's Science Undergraduate Laboratory Internship under the direction of Eric Charles at the Kavli Institute for Particle Astrophysics and Cosmology, Stanford Linear Accelerator Center.

Participant:

Signature

Research Advisor:

Signature 


\section{TABLE OF CONTENTS}

1 Introduction $\quad 1$

2 Materials and Methods $\quad 1$

2.1 Detector and Calibrations . . . . . . . . . . . . . . . . 1

2.2 Trending . . . . . . . . . . . . . . . . . . . . 4

2.3 Photoelectron Calculation ....................... 5

2.4 Veto Efficiency . . . . . . . . . . . . . . . . . . . . . . . . 6

$\begin{array}{llr}3 & \text { Results } & 7\end{array}$

3.1 Trending . . . . . . . . . . . . . . . . . . . . . . 7

3.2 Photoelectron Calculations . . . . . . . . . . . . . . . . . . 8

3.3 Veto Efficiency . . . . . . . . . . . . . . . . . . . . . 9

4 Conclusion $\quad 11$

5 Acknowledgments $\quad 11$

$\begin{array}{ll}\text { References } & 11\end{array}$ 


\begin{abstract}
Calibration analyses and Efficiency Studies for the Anti Coincidence Detector on the Fermi Gamma Ray Space Telescope. CHRIS KACHULIS (Yale University, New Haven, CT 06520) ERIC CHARLES (Kavli Institute for Particle Astrophysics and Cosmology, Stanford Linear Accelerator Center, Stanford, CA 94025)

The Anti Coincidence Detector (ACD) on the Fermi Gamma Ray Space Telescope provides charged particle rejection for the Large Area Telescope (LAT). We use two calibrations used by the ACD to conduct three studies on the performance of the ACD. We examine the trending of the calibrations to search for damage and find a timescale over which the calibrations can be considered reliable. We also calculated the number of photoelectrons counted by a PMT on the ACD from a normal proton. Third, we calculated the veto efficiencies of the ACD for two different veto settings. The trends of the calibrations exhibited no signs of damage, and indicated timescales of reliability for the calibrations of one to two years. The number of photoelectrons calculated ranged from 5 to 25. Large errors in the effect of the energy spectrum of the charged particles caused these values to have very large errors of around 60 percent. Finally, the veto efficiencies were found to be very high at both veto values, both for charged particles and for the lower energy backsplash spectrum.
\end{abstract}




\section{INTRODUCTION}

The Anti Coincidence Detector (ACD) on the Fermi Gamma Ray Space Telescope is a detector system built around the silicon strip tracker on the Large Area Telescope (LAT). The purpose of the ACD is to provide charged particle rejection for the LAT. To do this, the ACD must be calibrated correctly in flight, and must be able to efficiently veto charged particle events while minimizing false vetoes due to "backsplash" from photons in the calorimeter [1]. There are eleven calibrations used by the ACD. In this paper, we discuss the use of two of these calibrations to preform three studies on the performance of the ACD. The first study examines trending of the calibrations to check for possible hardware degradation. The second study uses the calibrations to explore the efficiency of an on-board hardware veto. The third study uses the calibrations to calculate the number of photoelectrons seen by each PMT when a minimum ionizing particle is detected, which is a useful value for performing simulations.

\section{MATERIALS AND METHODS}

\subsection{Detector and Calibrations}

The ACD is composed of eighty-nine $10 \mathrm{~mm}$ and $12 \mathrm{~mm}$ thick plastic scintillator tiles, which cover the top and four sides of the tracker. The top tiles are arranged in a five by five square, while the four sides have three rows of five tiles each above one large rectangular tile. The gaps between tiles are covered by eight $1.5 \mathrm{~mm}$ wide fiber scintillating ribbons. The ACD is shown from the side in Figure 1 [2]. Each tile is optically linked to two separate photo multiplier tubes (PMTs) by wavelength shifting fibers. Scintillation in the tiles from charged particles emits photons which are detected by the PMT's. The voltage readout from the PMT is then converted to a digital signal, called a Pulse Height Analysis (PHA) [1].

We wrote calibration programs in Python using the CERN data analysis package Py- 
ROOT [3]. For this paper we are interested particularly in two calibrations; the Pedestal and MIP Peak calibrations. The Pedestal calibration finds the PHA base signal for each PMT. The circuitry of the PMT increases the base voltage from zero, so even when there are no particles passing through the tiles, the voltage output is not zero, and so the PHA signal is also nonzero. Thus, the Pedestal calibration is finds the PHA signal that corresponds to no physics. This is done by taking measurements of the PHA on a periodic trigger at a rate of $2 \mathrm{~Hz}$. Since there is usually no physics in a particular tile, the resulting histogram will have a peak at the pedestal value which is nicely fit by a Gaussian. The mean of the Gaussian is then the value of the pedestal. We can deal with the small number of events where there are particles in the detector by confining the range over which the Gaussian is fitted. A typical pedestal fit is shown in Figure 2. As can be seen in Figure 2, the fitted pedestal value has a very low error, since the peak is very sharp. Typical values for the width of the Gaussian are around 3 PHA counts.

The MIP peak calibration finds the PHA signal of a minimum ionizing particle, which is known to correspond to about $1.9 \mathrm{MeV}$ at normal incidence in the $10 \mathrm{~mm}$ tiles. It is important to clarify what we mean when we refer to a MIP. Usually the term MIP refers to a particle at the minimum of the Bethe-Bloch curve. For protons passing through the $\mathrm{ACD}$, this minimum corresponds to a proton energy of $3.19 \mathrm{GeV}$. However, as can be seen in Figure 3, the geomagnetic cutoff ranges from about $6 \mathrm{GeV}$ to about $14 \mathrm{GeV}$ in Fermi's orbit. This means that lower energy particles, including protons that are usually called MIPs, will be swept away by the earth's magnetic field and will not reach the ACD. So when we refer to a MIP, we are referring to protons right around these geomagnetic cutoffs, since this are the protons that will deposit the lowest energy in the ACD. Because of the shallow rise in the Bethe-Bloch curve above minimum ionizing energies, the spread in the proton energies actually causes a very small spread in the mean energies deposited in the ACD, as shown in Figure 4. This spread is further diminished by the fact that the cosmic ray proton spectrum 
falls off very sharply as energy increases. So most of the particles measured by the ACD will deposit about the same mean energy. To find this peak, we select events where a track points to a tile. We then normalize for the angle of incidence and the width of the tile. Histograms of these normalized events contain two peaks, one at the MIP peak, and another backsplash peak, as shown in Figure 5.

The backsplash peak is actually due to three components, backsplash X-rays from the calorimeter, cosmic X-rays, and residual signal from previous events. We call this peak backsplash because the backsplash is the component of this spectrum which is important to this paper since backsplash is the component associated with physics events. The backsplash peak fits very well to an exponential decay.

The MIP peak (assuming a single mean energy value for incoming particles) is in theory a Landau distribution of the energy lost by a MIP in the tile convolved with a Poisson distribution of the PMT counts which has been stretched to account for the conversion from photoelectron counts to PHA counts (for further discussion see section 3.2, specifically Eq.5). However, since fitting a convolution is impractical, we must use other methods. We use three separate fits on the MIP peak, all of which give very consistent MIP peak values. The first fit is a Poisson distribution added to a decaying exponential to account for the backsplash. Since the Landau distribution is significantly thinner than the distribution of the MIP peak (see section 2.3 for calculation) a simple Poisson distribution works quite well. The Poisson distribution works particularly well at low PHA values, which makes it very useful for efficiency calculations since the vetoes we study are at low PHA values. The Poisson-Exponential fit is shown in Figure 6.

The second fit is a Gaussian, again added to a decaying exponential to account for the backsplash. This is a reasonable fit because when the expected value of a Poisson distribution is large it becomes similar to a Gaussian. As will be seen in section 3.2, the number of photoelectrons seen by the PMT's ranges 5 to 25, which is right in the area 
where it becomes reasonable to estimate a Poisson distribution with a Gaussian. For this reason, the Gaussian cannot be trusted at low PHA, which is where it diverges most from the Poisson-Landau convolution, and thus where the Poisson distribution is necessary. However, the width and mean of the Gaussian are still trustworthy, which is important. The Gaussian has an advantage over the Poisson because the Gaussian's width is not defined as the (scaled ) square root of its mean. Thus the Gaussian can allow for the combination of the spread from the counting statistics of the PMTs, the Landau distribution, and the particle energy spectrum. This allows us to separate the different contributions, which is important when calculating the number of photoelectrons counted by each PMT. The Gaussian-Exponential fit is shown in Figure 7.

The final fit is a double Gaussian. This fit is used to fit higher PHA values than the other two fits. At higher PHA values the Poisson-Landau convolution falls below the histogram because of contamination from higher energy particles. The double Gaussian allows us to extend our fit to higher values and the calculate more accurate values for the total number of charged particle events, which we need to know to calculate veto efficiencies. Above the range of the double Gaussian we simply add up the number of events in the histogram. The double Gaussian fit is shown in Figure 8

\subsection{Trending}

We check for possible signs of degrading due to radiation damage that is a danger for any detector in space by examining the trends of MIP peak and Pedestal calibrations. We would expect degrading of the tiles or the optical connections to cause systematic downward trending in MIP peak values, while leaving Pedestal values unchanged. Degrading of the PMT and Analog to Digital Converter (ADC) circuitry could cause a the Pedestal values to trend either up or down, depending on the manner in which the circuitry is degrading. To examine this trending, we sampled data from once every four weeks between weeks 28 and 
108 of the mission. We then plotted Pedestal and MIP peak values versus time, and fit a straight line to the data. The slope of that line indicates the trend of the calibration.

\subsection{Photoelectron Calculation}

We use the Exponential-Gaussian fit to calculate the number of photons seen by each PMT. The spread of the MIP peak is due to three sources; the counting statistics which follow a Poisson distribution with standard deviation $\sqrt{N}$ where $N$ is the number of counts, the Landau distribution of the energy lost by a particle in the scintillator, and the particle energy spectrum. The Landau distribution has been well studied, and it is known that the full-width-at-half-maximum of the Landau distribution is $4 \xi$ where:

$$
\xi=\frac{K}{2 N_{A}} \frac{n_{e} w}{\beta^{2}}
$$

for $\mathrm{K}=0.307075 \mathrm{MeV} \mathrm{cm}{ }^{2} \mathrm{~mol}^{-1}, N_{A}$ Avogadro's number, $n_{e}$ the electron density of the absorber, and $w$ the width of the absorber[4]. We normalized our events to a tile width of $1 \mathrm{~cm}$, and the ACD tiles are EJ-200 Plastic Scintillators manufactured by Eljen Technology [5]. The electron density of the scintillators is specified as $n_{e}=3.33 \times 10^{23}$ electrons $/ \mathrm{cm}^{3}$ [6]. Our particle spectrum is dominated by particles right around the geomagnetic cutoff, which ranges from about $8 \mathrm{GeV}$ to about $16 \mathrm{GeV}$ over the path of the satellite. These correspond to beta values for protons of $\beta=0.993$ and $\beta=0.998$. Plugging everything in and using the average of our two $\beta$ s, we find that $\xi=0.0857 \mathrm{MeV}$. This indicates a f.w.h.m. of the Landau distribution of $0.344 \mathrm{MeV}$. The f.w.h.m. of the MIP peak is generally around $1.5 \mathrm{MeV}$, thus indicating that the spread of the Gaussian is due mainly to the Poisson counting statistics. This explains why the Exponential-Poisson fit works well. The contribution from the particle energy spectrum has not been well studied. To get a very rough estimate we assume that this spread contributes a spread of 12 percent of the MIP peak. It should be noted that this 
is an extremely rough estimate. The energy loss spectrum is likely not particularly Gaussian, so it's effect on the spread will need to be very well studied to be correctly accounted for. We then subtract both this spread and the spread due to the Landau from the Gaussian spread in quadrature.

$$
\sigma_{C}=\sqrt{\sigma^{2}-\sigma_{L}^{2}-\sigma_{E}^{2}}
$$

where $\sigma_{C}$ is the standard deviation due to counting statistics, $\sigma_{L}$ is from the Landau distribution, $\sigma_{E}$ is from the particle energy spectrum and $\sigma$ is the original standard deviation of the fit. Then, from the square root law, we know that:

$$
\frac{\mu}{\sigma_{C}}=\frac{N}{\sqrt{N}}=\sqrt{N}
$$

where $\mu$ is the mean of the Gaussian. Finally, since the PHAs are normalized for the path length of the particle through the $\mathrm{ACD}$, we divide our calculated number of photoelectrons by the average path length factor (defined as the path length divided by $10 \mathrm{~cm}$ ) to find the number of photoelectrons counted by each PMT for a MIP at normal incidence.

One complication that must be accounted for is that because of the asymmetry of the Landau distribution, the convolution of the Landau with the Poisson shifts the peak about 5 percent higher. So in practice we use the formula:

$$
\frac{0.95 \mu}{\sigma_{C}}=\frac{N}{\sqrt{N}}=\sqrt{N}
$$

\subsection{Veto Efficiency}

We used the Exponential-Poisson distribution to study the efficiency of the onboard hardware veto. As explained above in sections 2.1, the exponential in this fit accounts for the backsplash peak. One of the objectives of the ACD is to avoid falsely vetoing backsplash events,

which is one of the three components of the backsplash peak [5]. The Poisson distribution 
accounts for the charged particles we want to veto. We can thus separate the histogram into these two components, and analyze the respective fraction of charged particles and backsplash rejected by a particular veto setting.

We also use the Double Gaussian fit when measuring the efficiency. The ExponentialPoisson fit allows us to separate the different components around the first dip, where they both have noticeable effects. The Double Gaussian is used at higher PHA to allow us to extend the fit farther than the reasonable range of the Exponential-Poisson fit, and more closely match the higher energy particle contamination. These two fits are stitched together just above the peak, as shown in Figure 9. Beyond the range of the Double Gaussian a simple count of the number of entries in the histogram is used. It is important to note that the histograms cut off around 2000 PHA. Around 15 percent of the total events are generally above 2000 PHA, so the total number of charged particles must be multiplied by 1.15 before being used for efficiency calculations.

\section{RESULTS}

\subsection{Trending}

Neither the MIP peak nor the Pedestal trends exhibit any evidence of systematic drift. Histograms of these trends are shown in Figure 10 and Figure 11. As can be seen from these histograms there is no systematic drift in either calibration. Since the MIP peak values would be expected to trend downward if tile or optical connection damage were beginning to affect them, the Gaussian distribution of the MIP peak trend indicates that the possible trends of individual PMTs are more likely statistical noise than actual degrading of individual tiles or PMT optical connections. This indicates there is not yet a noticeable effect from radiation damage to the tiles or optical connections.

The same logic cannot be applied to the Pedestal trends, since these would be expected 
to trend both up and down, depending on the particular damage in each PMTs respective circuitry. However, the sharpness of the Pedestal trend peak around zero indicates that the Pedestal values have been remaining very stable, and thus give no indication of any degrading of the circuitry.

The stability of the calibrations indicates that at current rates the calibrations can be trusted for at least a couple of years. For the pedestal calibration, a two sigma tile calibration will be within 5 percent of correct for 3.74 years. For the MIP peak calibration, it will be within 5 percent for 1.80 years. This does not mean that current calibrations should be taken as correct for the next two years, since future damage to the instrument could greatly change calibrations. It only indicates that no significant damage has yet occurred, and that calibrations from a few years ago can still be considered reasonably valid.

\subsection{Photoelectron Calculations}

The number of photoelectrons counted by each PMT from a MIP at normal incidence was calculated for run 0299978033. The run number indicates that the data run began at 23:13:53 UTC on July 4, 2010. Figure 12 shows a histogram of the number of photoelectrons counted by each PMT. Two PMTs (PMT A on tile 220 and PMT A on tile 423) are not included in the histogram because they have poor optical connections which cause their event histograms to be too skewed to make reliable fits. The number of photoelectrons ranges from just above around 5 to around 26 photoelectrons, with a mean of 11.87 photoelectrons. The errors on these values are very large (around 60 percent) because of the large uncertainty of the particle energy spectrum effect. In order to make more reliable calculations, the effect of the particle energy spectrum must be further studied and well understood.

We can use our photon calculations to calculate the theoretical distribution of PHA values that they should result in and compare it to the actual histogram of events. In doing this, we ignored the effects of the particle energy spectrum since we do not understand it very well. 
The Landau distribution is computed easily in ROOT from the MIP peak value (multiplied 0.95 to account for the shift caused by the convolution) and the value of $\xi$ calculated above. We normalized this distribution empirically for the number of particles in the histogram which are MIPs (not backsplash peak or higher energy contamination). The calculated photoelectron value gives us a photoelectron PHA ratio, $\rho_{p}=\frac{\mu}{N_{p}}$. The distribution is then calculated point by point as:

$$
D(x)=\int_{-\infty}^{\infty} P_{\rho_{p}, \frac{x^{\prime}}{\rho_{p}}}(x) L\left(x^{\prime}\right) d x^{\prime}
$$

where $P_{\rho_{p}, \frac{x^{\prime}}{\rho_{p}}}$ is the Poisson distribution with mean $\frac{x^{\prime}}{\rho_{p}}$ stretched by a factor of $\rho_{p}$. This Poisson distribution is thus a PHA version of the regular Poisson distribution with a mean of $\mathrm{x}$ '. We calculated the Poisson-Landau distribution $\mathrm{D}(\mathrm{x})$ as a discrete sum with step size 1 and limits $x \pm 1000$. Figure 13 shows the resulting distribution in red along with the corresponding histogram of events. As can be seen in Figure 13, the Poisson-Landau fits very well to the histogram of events. This may indicate that the particle energy spectrum effect is rather small, although further study is needed to draw any real conclusions.

Figure 14 compares the Poisson-Landau distribution to the Exponential-Poisson and Exponential-Gaussian fits in the low PHA range where the vetoes are studied. As can be seen in Figure 14 the Exponential-Poisson matches the actual Poisson-Landau distribution much better than the Exponential Gaussian in this range. This is the reason we use the Exponential-Poisson fit when studying the veto efficiency.

\subsection{Veto Efficiency}

We calculated the charged particle and backsplash efficiencies for run 0299978033 for different vetoes ranging from 0.2 MIPs to 0.6 MIPs. For the charged particles, efficiency was defined as the fraction of events rejected by a particular veto, while for the backsplash efficiency was 
defined as the fraction of events allowed through by a particular veto. So, for both elements higher efficiencies are better.

Because of the setup of of the ACD cuts, we are particularly interested in the veto efficiencies at $0.22 \mathrm{MIPs}$ and $0.44 \mathrm{MIPs}$. In the LAT photon selection process there is one cut which examines the ratio of the energy in the ACD to the energy in the calorimeter and rejects the event if this ratio is greater than 0.008 . Thus this cut will reject a $100 \mathrm{MeV}$ event if any tile records over 0.44 MIPs. There is a tighter cut at $0.22 \mathrm{MIPs}$ when a track points directly to within $16 \mathrm{~mm}$ of the ACD tile where energy is recorded, since such an event is less likely to be backsplash.

Histograms of the charged particle and backsplash efficiencies for a veto of 0.44 MIPs are shown in Figures 15 and 16. Again the two bad PMTs are not included. As can be seen in Figure 15, the average charged particle efficiency at 0.44 MIPs is 0.988 with a standard deviation is 0.00765 . The average backsplash efficiency is slightly lower, at 0.9438 . The backsplash efficiencies have a wider spread than the charged particle efficiencies, with a standard deviation of 0.02002 . This is partly due to the fact that since the charged particle efficiencies are closer to 1, the spread, particularly on the high side, gets squeezed together more simply by the fact that the efficiency cannot be greater than 1 . This effect is also responsible for the fact that the backsplash distribution is more symmetric than the charged particle distribution.

Since an event with a track pointing all the way to the ACD is less likely to be backsplash, we are more concerned with the charged particle efficiency for the 0.22 MIP cut than with the backsplash efficiency. A histogram of the charged particle efficiency for the 0.22 MIP cut is shown in Figure 17. As can be seen the mean efficiency is 0.9992, with a standard deviation of 0.001352 . 


\section{CONCLUSION}

We used the pedestal and MIP peak calibrations for the ACD on the Fermi LAT to conduct three studies. In the first study, an examination of the trends of the calibrations revealed no indication of degrading hardware on the LAT. We calculated timescales over which the pedestal and MIP peak calibrations would remain reliable. The pedestal calibration remains reliable for a timescale of about 3.74 years, while the MIP peak calibration remains reliable for a timescale of about 1.8 years. In the second study we measured the efficiencies of different veto thresholds, with particular interest in the efficiencies at 0.44 MIPs and 0.22 MIPs. The charged particle efficiency was found to be on average 0.9992 at 0.22 MIPs and 0.988 at 0.44 MIPs while the backsplash efficiency was found to be 0.9438 at 0.44 MIPs. These high efficiencies indicate that the choices of those setting for ACD veto cuts are good decisions. Finally we made rough estimates of the number of photons seen by a PMT from a MIP at normal incidence. The mean of this calculation was 11.87 photons, although the large uncertainties of the effects of particle energy spectrum cause large uncertainties of around 60 percent in these photon calculations. In order calculate more precise values for the number of photons, the particle energy spectrum will need to be closely studied.

\section{ACKNOWLEDGMENTS}

I would like to thank my mentor Eric Charles for his guidance and support. I would also like to thank the U.S. Department of Energy and their financial support, SLAC for hosting the SULI program, and everyone involved in running the SULI program at SLAC for making the program run so smoothly. 


\section{REFERENCES}

[1] A. Moiseev et al., "The anti-coincidence detector for the glast large area telescope," Astroparticle Physics, vol. 27, pp. 339-358, 2007.

[2] http://fermi.gsfc.nasa.gov/acd/images/djt/ACD_June4.html, August 2010.

[3] "How to use the python pyroot interpreter?" http://root.cern.ch/drupal/content/ how-use-use-python-pyroot-interpreter, August 2010.

[4] C. Amsler et al., "Review of particle physics," Physics Letters, vol. B667, pp. 1+, 2008.

[5] A. Moiseev et al., "High efficiency plastic scintillator detector with wavelength-shifting fiber readout for the glast large area telescope," Nuclear Instruments and Methods in Physics Research A, vol. 583, pp. 372-382, 2007.

[6] EJ-200 PLASTIC SCINTILLATOR, Eljen Technology. 


\section{FIGURES}

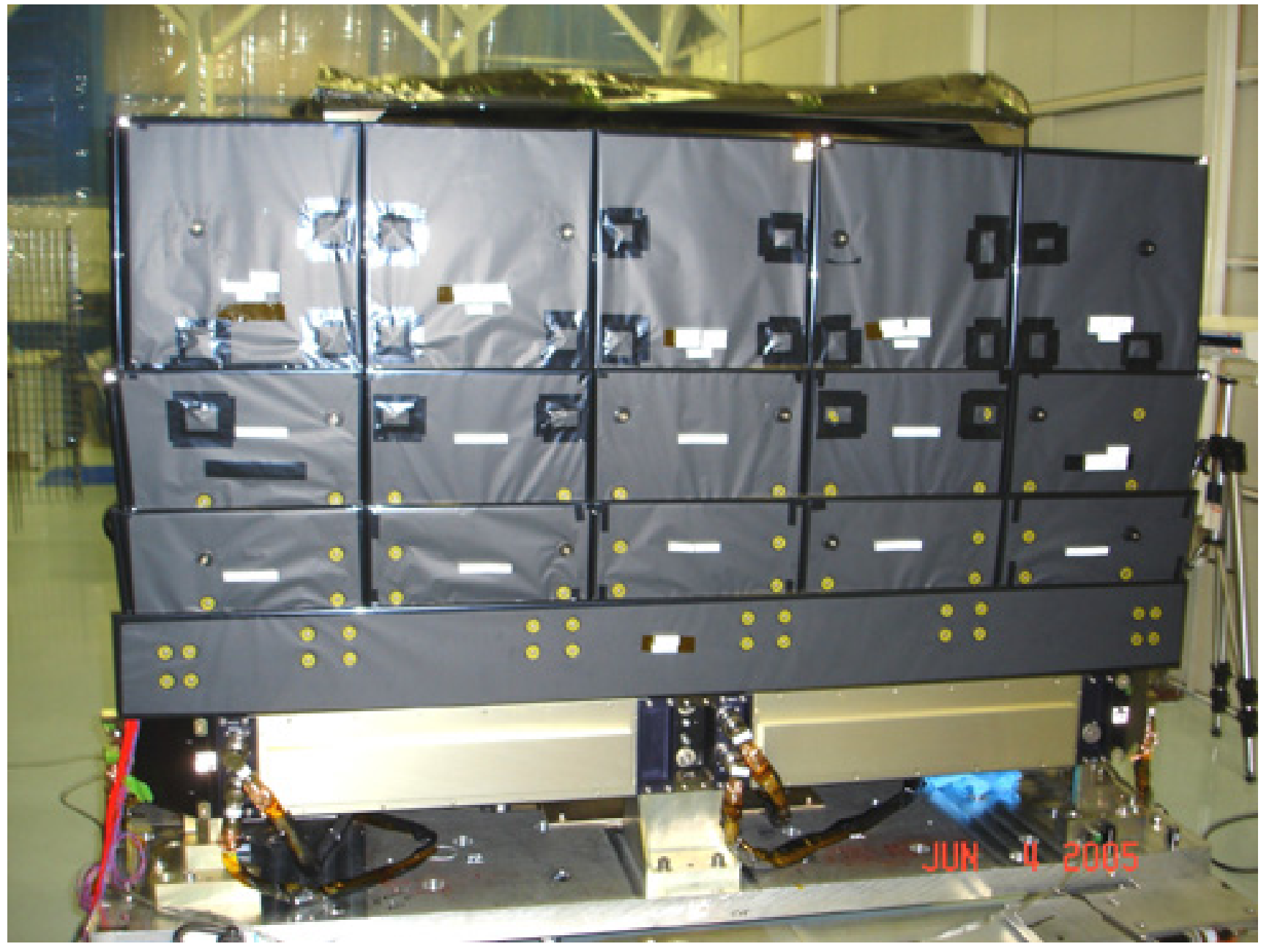

Figure 1: The ACD seen from the side. 


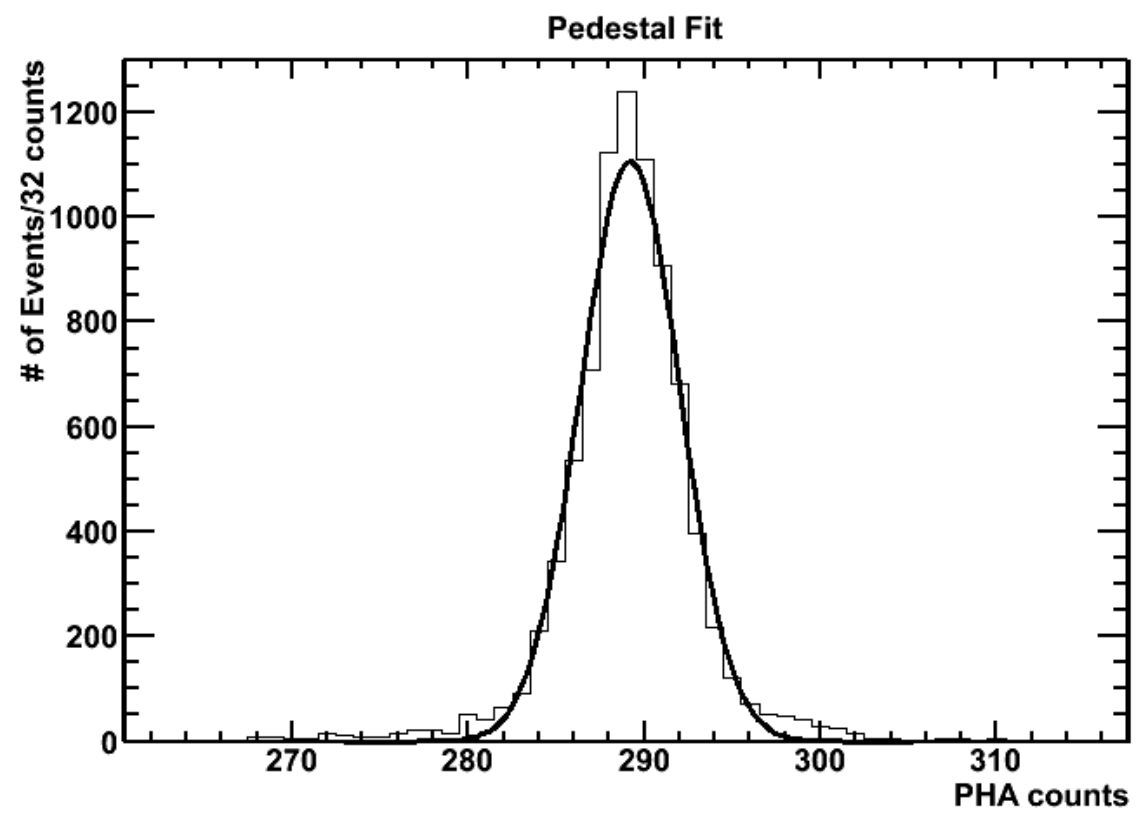

Figure 2: Typical Pedestal Fit
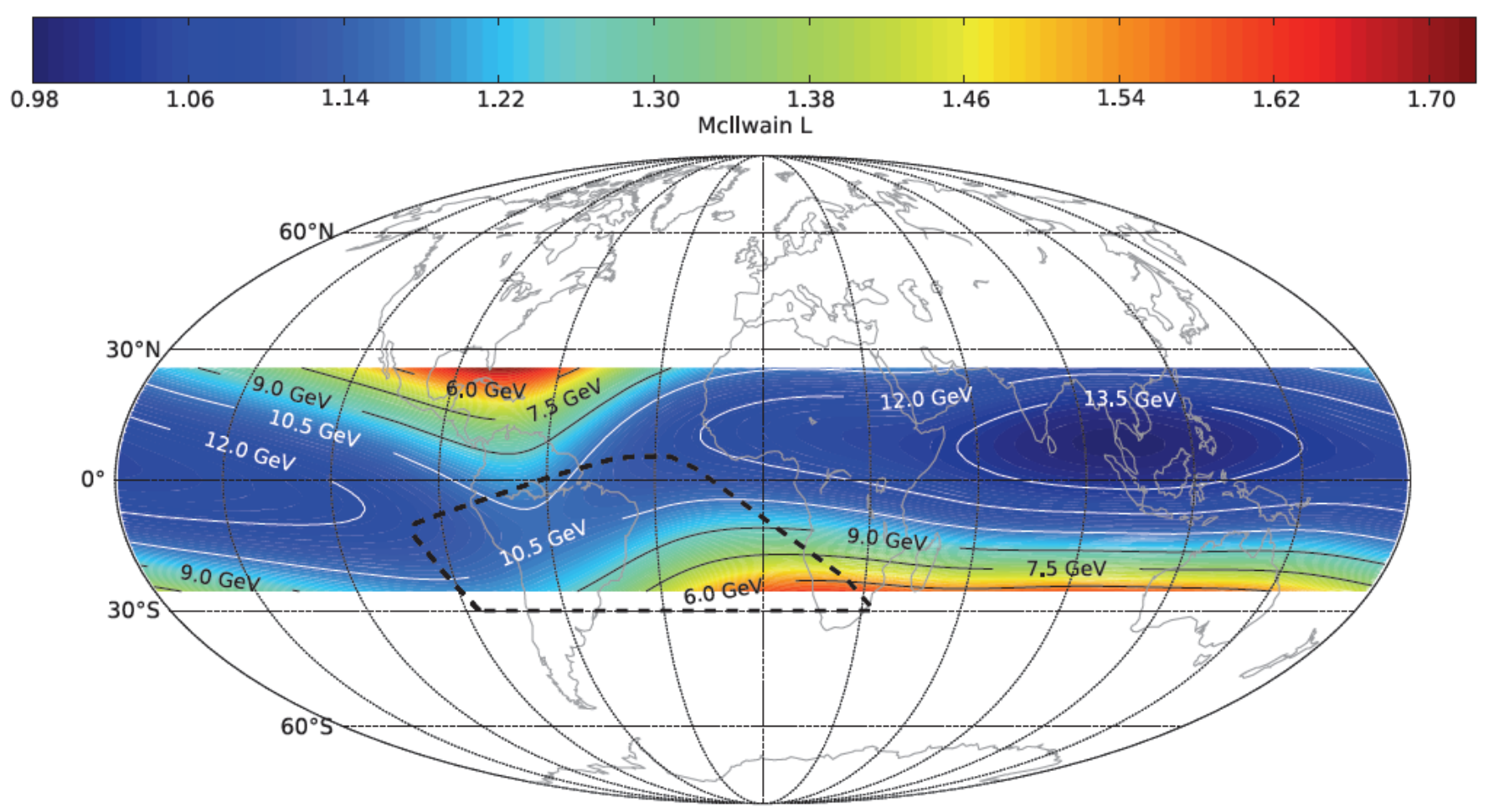

Figure 3: The geomagnetic cutoff in the orbit of Fermi, at Fermi's altitude. 


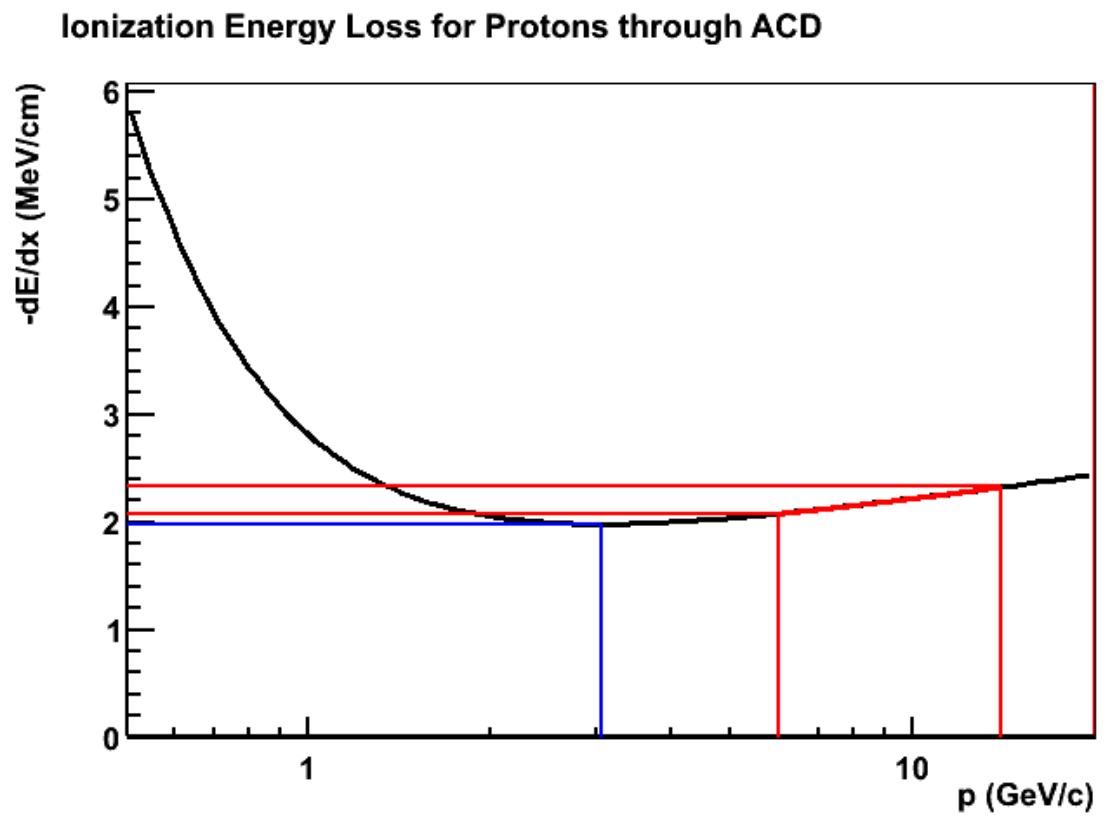

Figure 4: The Bethe-Bloch curve for particles in the ACD. The blue lines indicate a true "minimum ionizing particle." The red lines indicate the geomagnetic cutoff range over the orbit of Fermi.

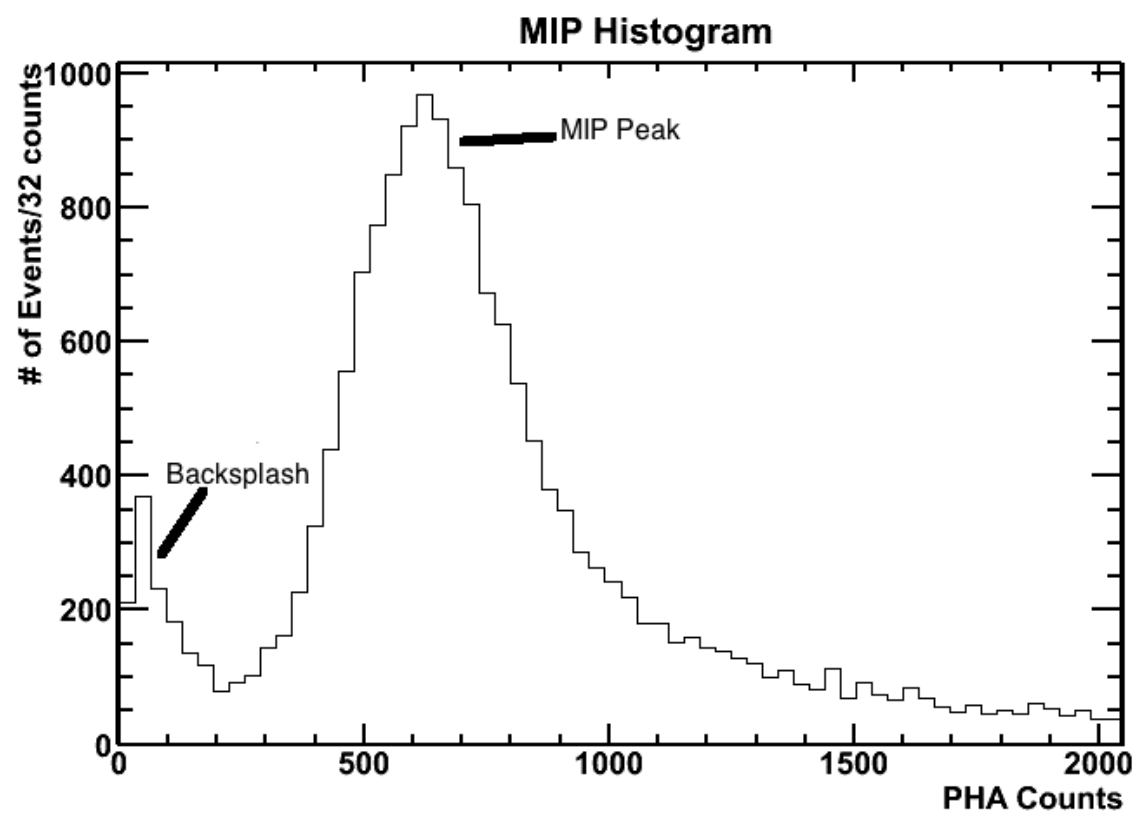

Figure 5: The two peaks of a MIP peak histogram 


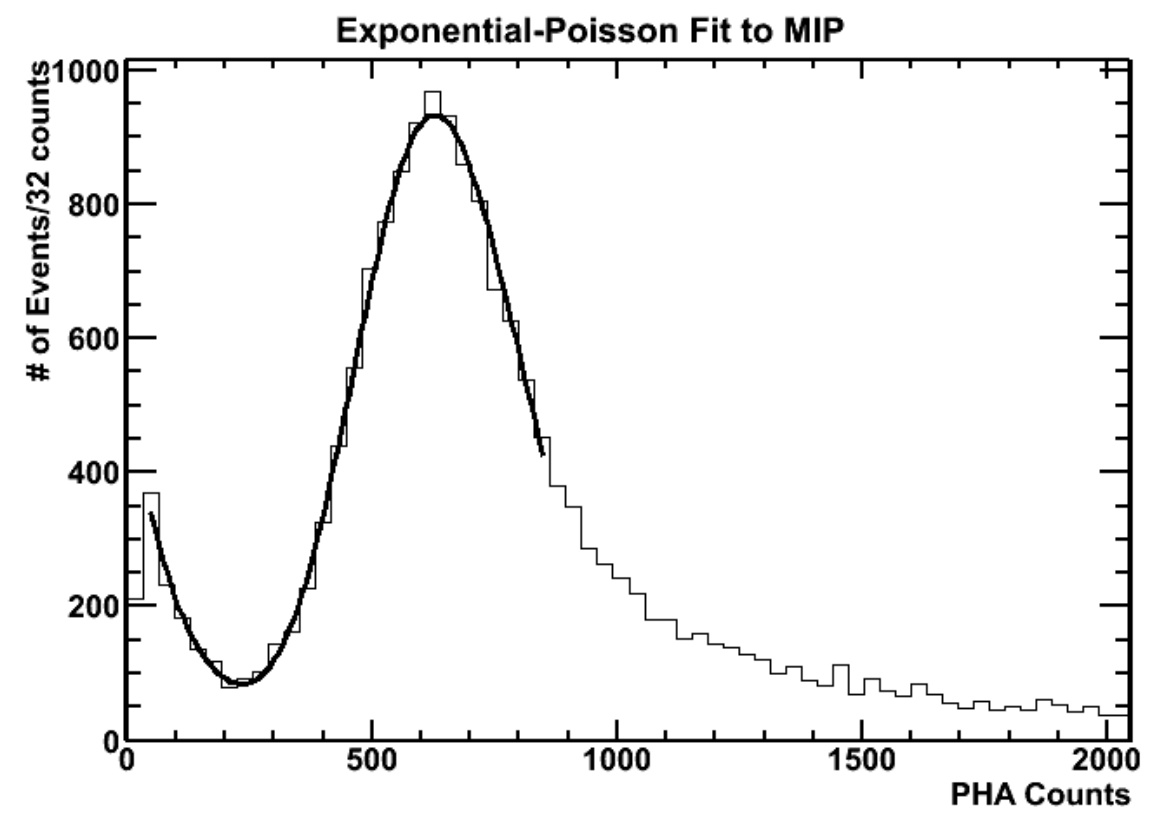

Figure 6: Exponential-Poisson fit to MIP peak.

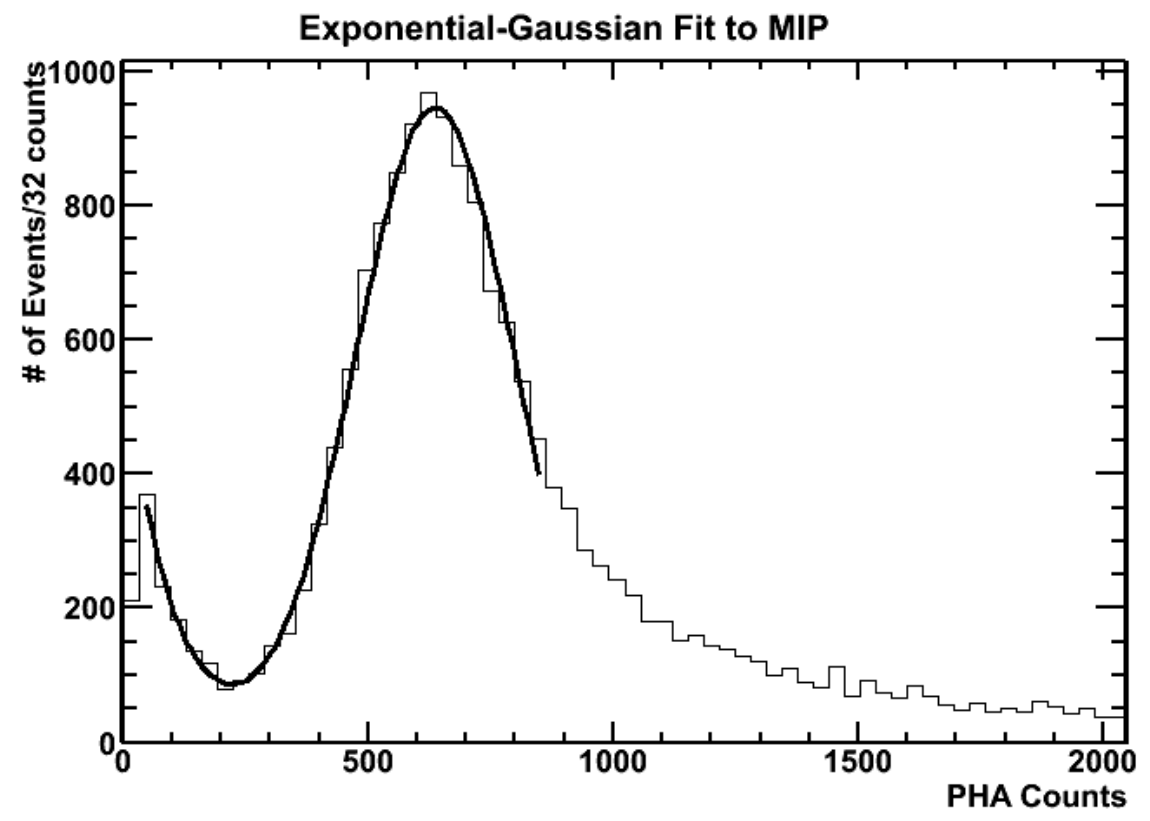

Figure 7: Exponential-Gaussian fit to MIP peak. 


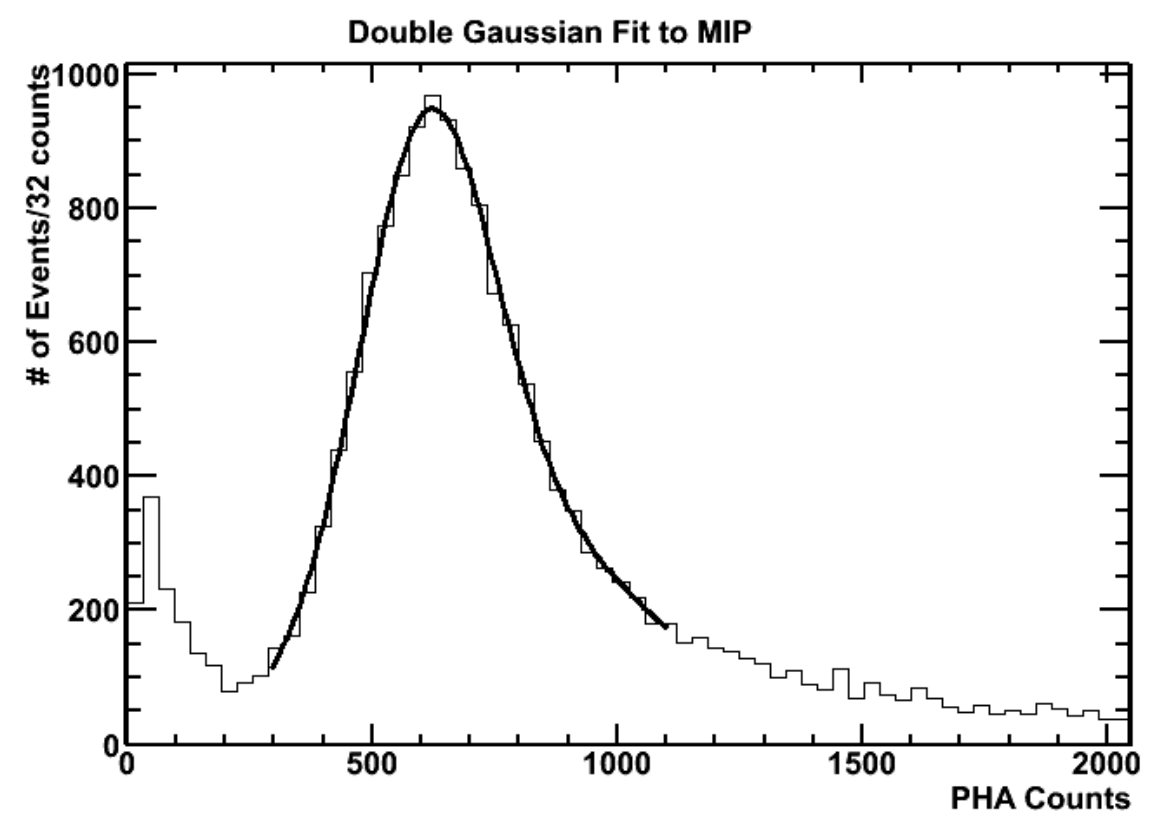

Figure 8: Double Gaussian fit to MIP peak.

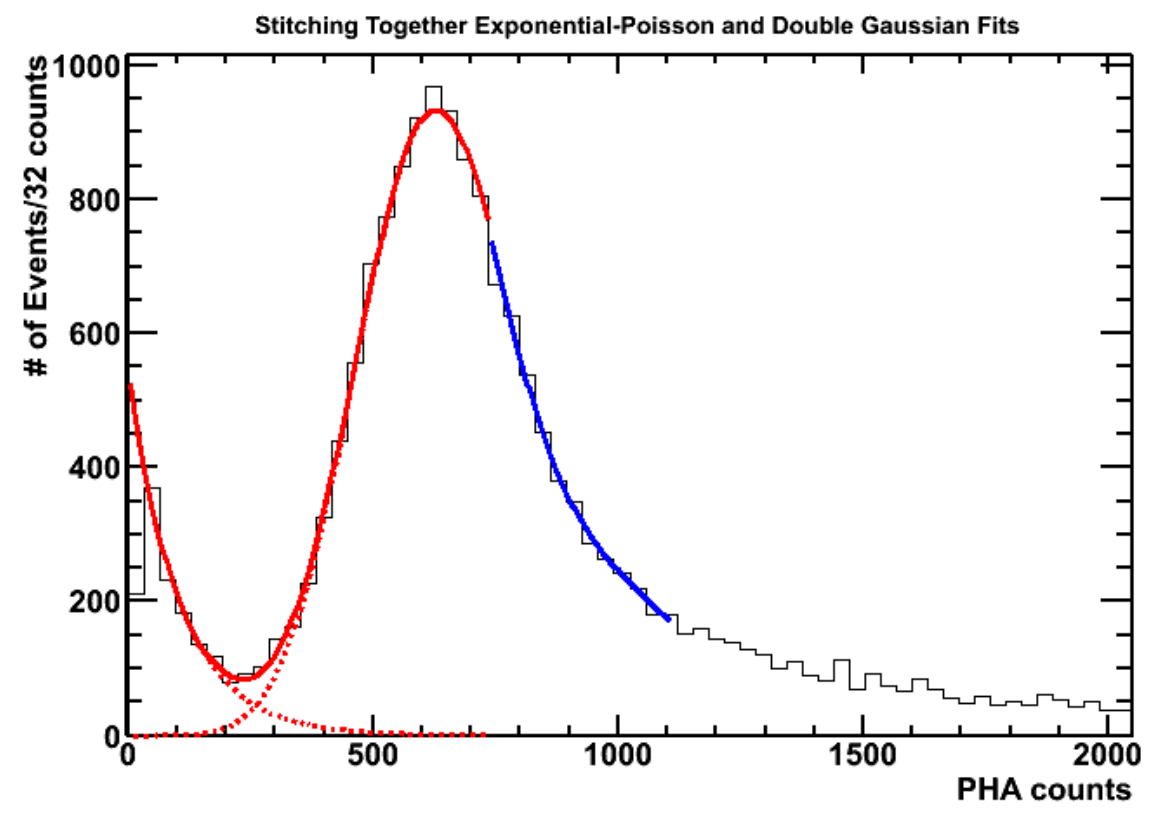

Figure 9: Stitching together the Exponential-Poisson and Double Gaussian fits. The red is the Exponential-Poisson fit, the blue is the Double Gaussian fit. The dotted red lines show how the histograms are separated into backsplash and charged particle components. 


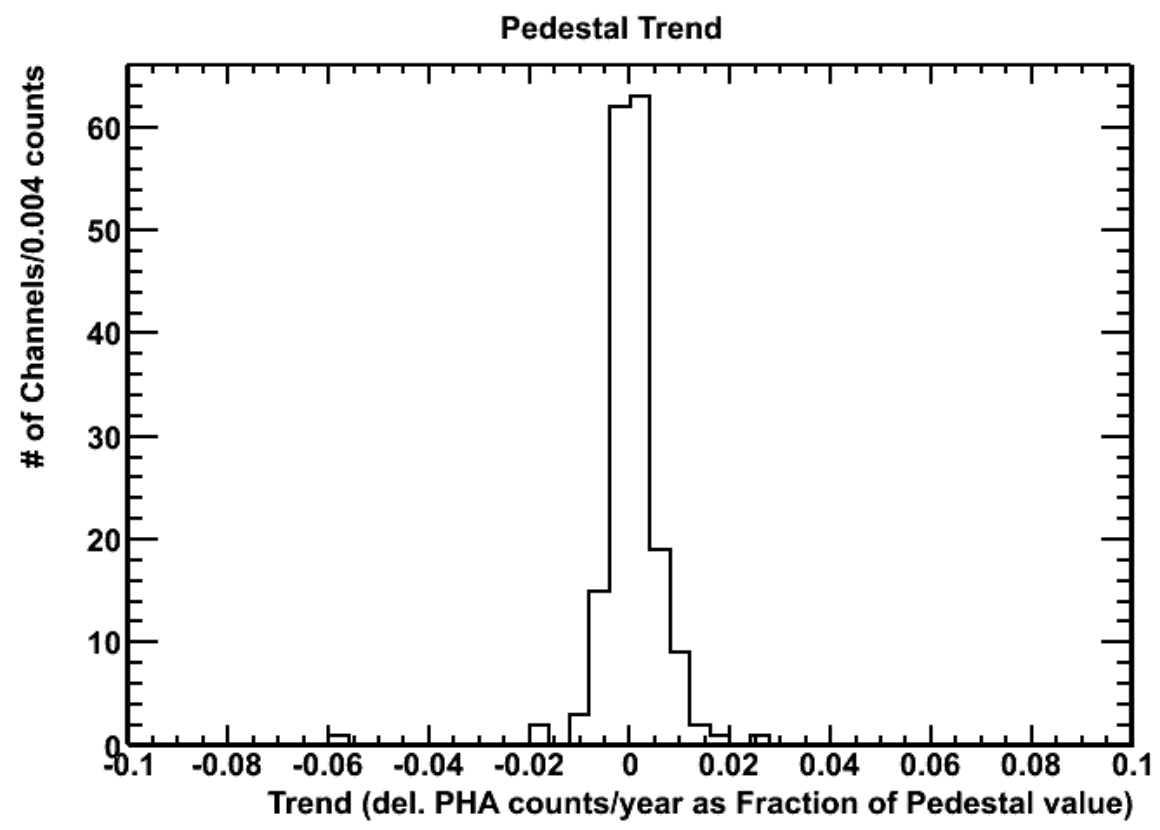

Figure 10: Histogram of Pedestal Trend

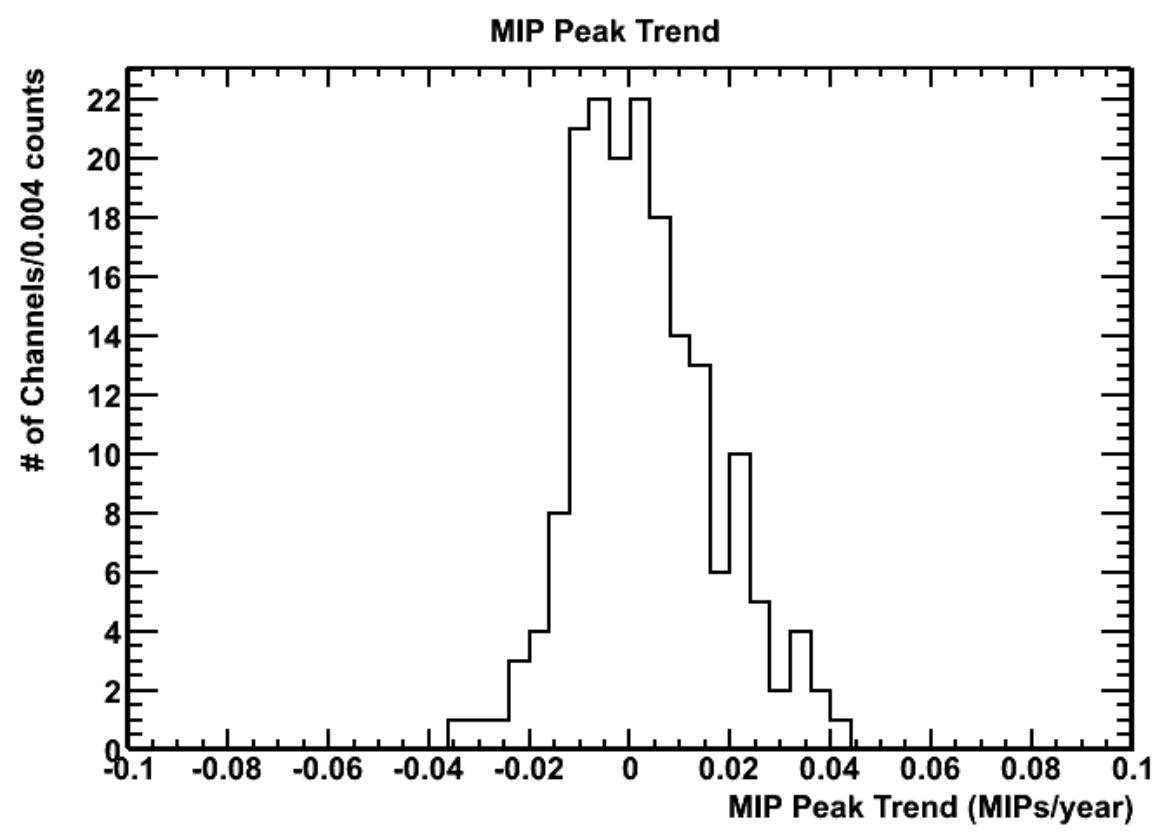

Figure 11: Histogram of MIP Peak Trend 
Photons Counted by a PMT for a MIP

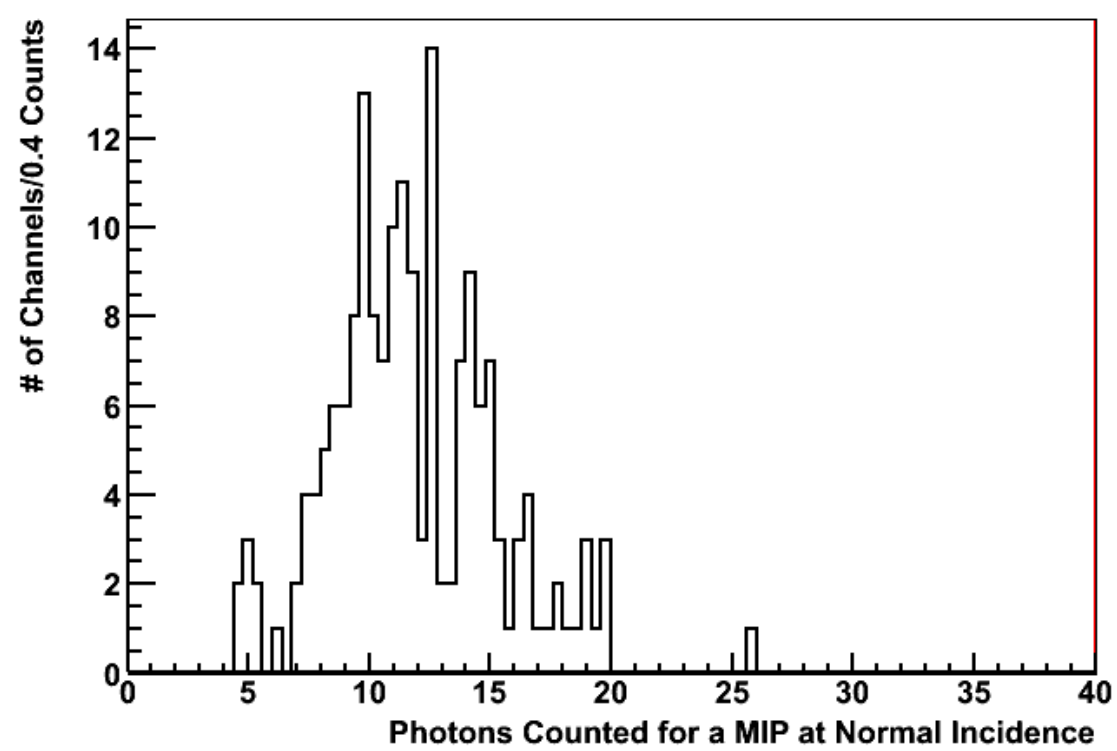

Figure 12: Photons seen for a MIP.

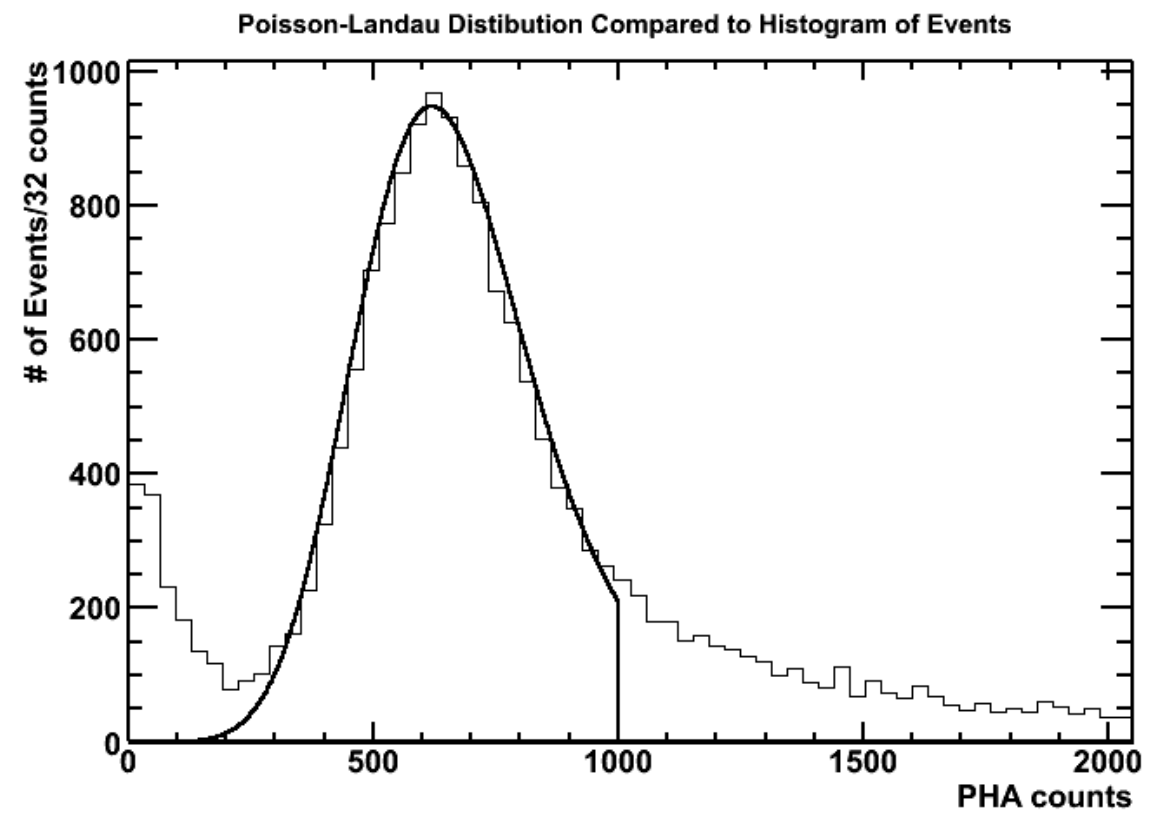

Figure 13: Comparison to Poisson-Landau distribution to histogram of events. 


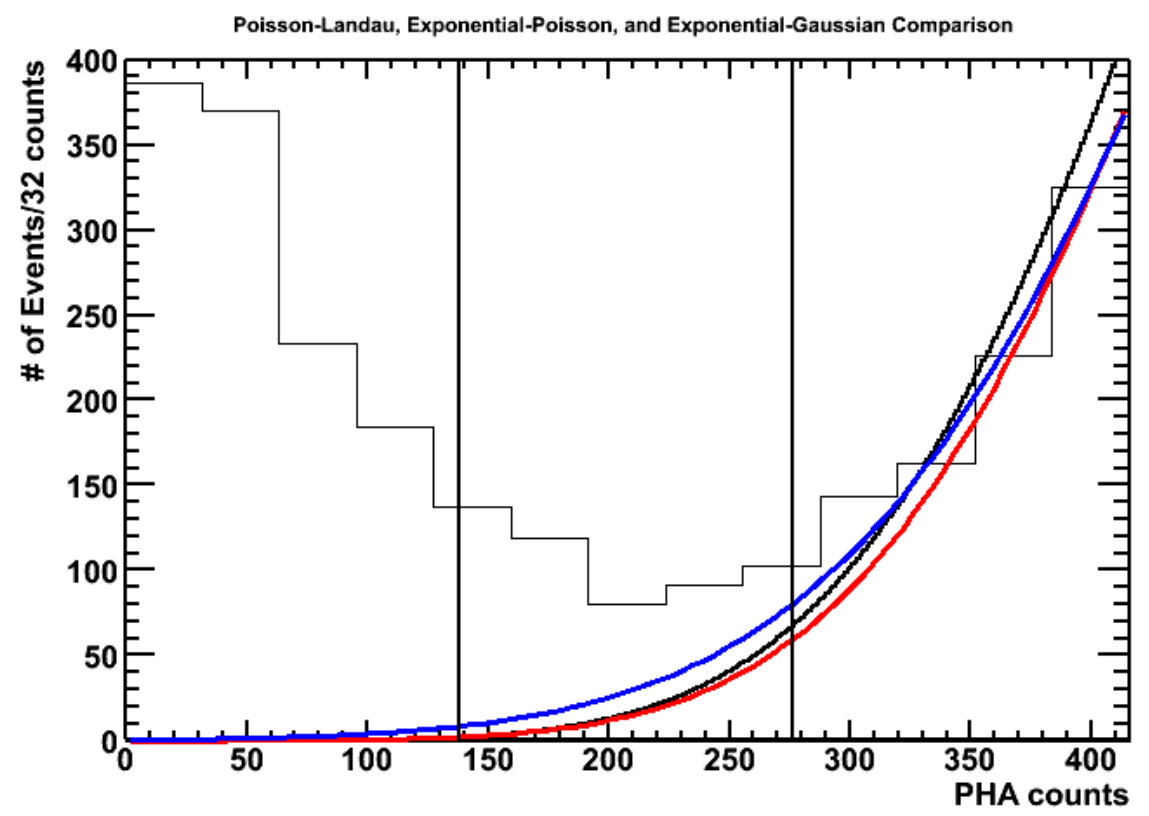

Figure 14: Comparison of Poisson-Landau convolution to Exponential-Poisson and Exponential Gaussian fits. The Poisson-Landau is black, the Exponential-Poisson blue, and the Exponential-Gaussian is red. The two vertical lines are at 0.22 MIPs and 0.44 MIPs.

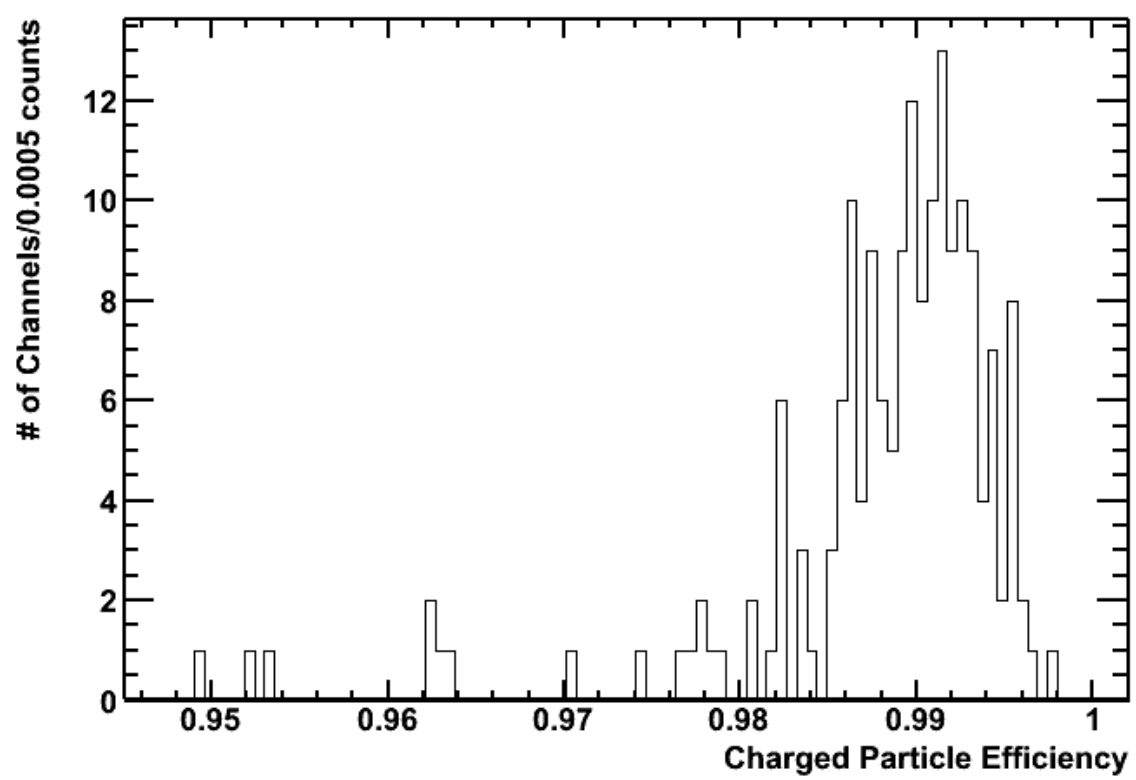

Figure 15: Histogram of charged particle veto efficiency at 0.44 MIPs 


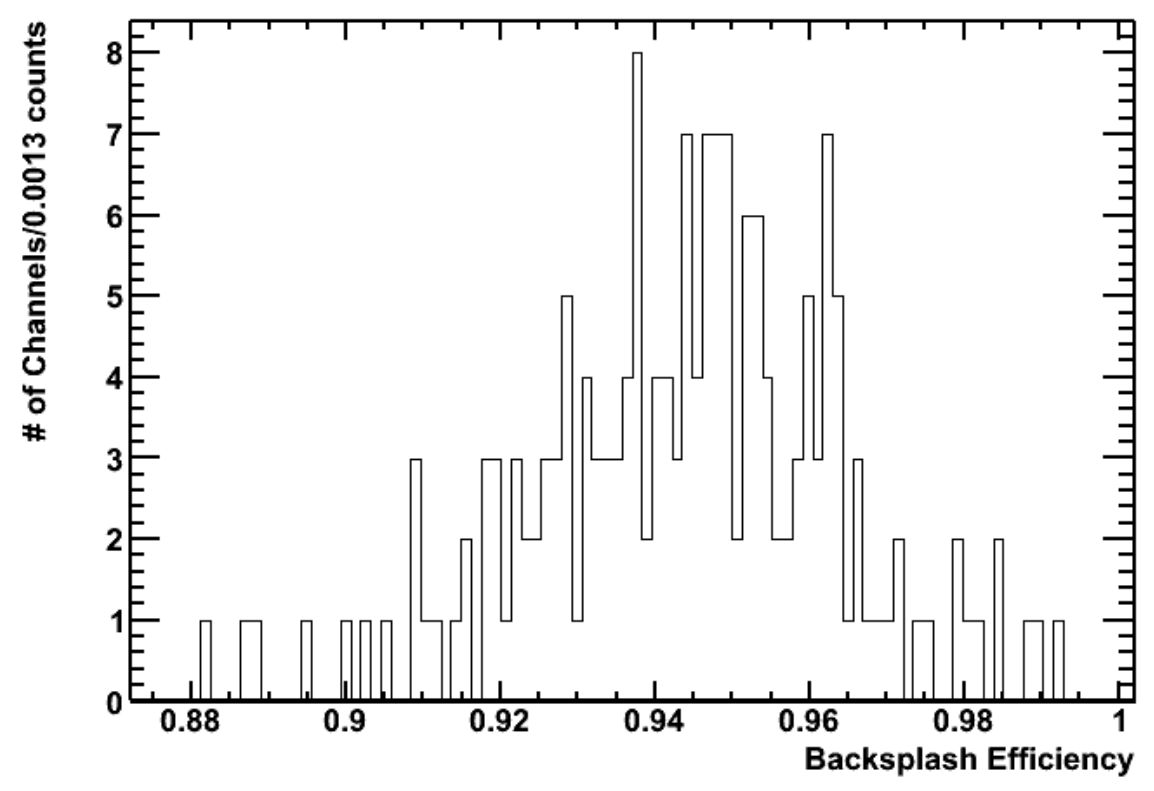

Figure 16: Histogram of backsplash veto efficiency at 0.44 MIPs

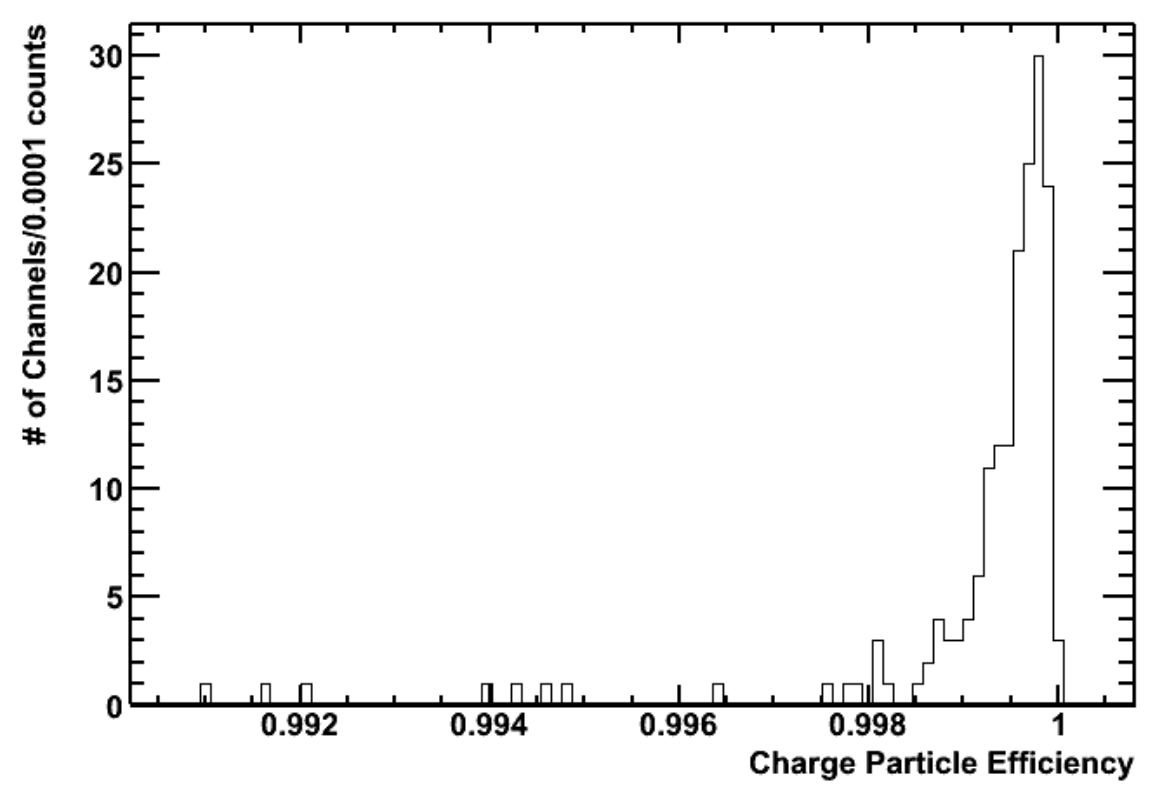

Figure 17: Histogram of charged particle veto efficiency at 0.22 MIPs 